\title{
Acute treatment optimization for migraine: results of the American migraine prevalence and prevention (AMPP) study
}

\author{
RB Lipton ${ }^{1 *}$, AN Manack², D Serrano ${ }^{3}$, DC Buse ${ }^{4}$ \\ From The European Headache and Migraine Trust International Congress \\ London, UK. 20-23 September 2012
}

\section{Objectives}

To assess and compare acute treatment optimization as measured by the Migraine Treatment Optimization Questionnaire (M-TOQ) within a population-based sample of persons with migraine.

\section{Methods}

AMPP is a longitudinal, US-population-based study for which questionnaires were mailed to 24,000 severe headache sufferers and followed annually. Respondents with ICHD- 2 migraine were stratified as either CM $(>15$ headache-days/month) or EM ( $<15$ headache-days/month). Acute-treatment optimization was measured with $\mathrm{M}$ TOQ, a valid/reliable patient-report tool assessing 5 domains: functioning, rapid relief, relief consistency, recurrence risk, tolerability over preceding 4 weeks. Respondents rated statements in each area as either occurring: never, rarely, $<$ or $>$ half the time. An item response theory (IRT) model used to define scaled treatment optimization scores as function of M-TOQ item set: lower scores=less/problematic optimization; higher scores=greater optimization. The model was expanded to incorporate persons with CM/EM on scaled scores and explored demographic adjustments for age and gender.

\section{Results}

8612 persons met criteria for migraine $(\mathrm{CM}=539$; $E M=8073)$ and completed M-TOQ. IRT model parameters indicated excellent M-TOQ psychometric properties. Scaled treatment optimization scores were significantly lower for persons with CM (3.25) vs EM (4.01, $\mathrm{b}=-0.757 ; \mathrm{p}<.0001)$, corresponding to a 0.5 standard deviation (SD) difference between CM and EM. After adjustment, mean difference on scaled-optimization score remained significantly lower (worse) for $C M(b=-0.751$; $\mathrm{p}<.0001)$.

\section{Discussion}

Treatment regimens were less well-optimized and more lacking in domains measured by M-TOQ (ie, functioning, rapid relief, consistency of relief, risk of recurrence and tolerability) among persons with CM vs EM. Funding: The AMPP study was funded through a research grant to the NHF from Ortho-McNeil Neurologics. Additional analyses were supported by Allergan, Inc.

\section{Author details}

'Department of Neurology, Montefiore Medical Center, Bronx, New York, USA. ${ }^{2}$ Allergan, Inc., Irvine, California, USA. ${ }^{3}$ Vedanta Research, Chapel Hill, North Carolina, UK. ${ }^{4}$ Department of Neurology, Montefiore Medical Center, Bronx, New York, UK.

Published: 21 February 2013

doi:10.1186/1129-2377-14-S1-P201

Cite this article as: Lipton et al: Acute treatment optimization for migraine: results of the American migraine prevalence and prevention (AMPP) study. The Journal of Headache and Pain 2013 14(Suppl 1):P201. 